\title{
A Negative Association between Melanin-Based Plumage Color Heterogeneity and Intensity in Birds
}

\author{
Ismael Galván ${ }^{*}$ \\ Sol Rodríguez-Martínez \\ Departamento de Ecología Evolutiva, Estación Biológica de \\ Doñana, Consejo Superior de Investigaciones Científicas, \\ Calle Américo Vespucio 26, 41092 Sevilla, Spain
}

Accepted 9/19/2018; Electronically Published 3/1/2019

Online enhancement: supplemental file.

\begin{abstract}
Even though plumage diversity is one of the most diverse phenotypic traits in nature, the reasons why some species exhibit more distinctive colors than others are poorly known. In the case of melanins, the most abundant pigments in birds, different chemical forms lead to different plumage colors and different amounts of those forms lead to different color intensities. However, the synthesis of some melanin forms is more physiologically limited than others. We hypothesize that an evolutionary solution to this scenario may consist in a negative association between melanin-based color heterogeneity and intensity. Here we confirm this prediction after analyzing the diversity and expression level of melanin-based plumage colors in 96 species of birds breeding in the Iberian Peninsula. After controlling for phylogenetic effects, the intensity of the plumage colors of birds decreased with the number of different colors, suggesting that the physiological mechanism of melanin synthesis does not favor the production of both a heterogeneity of melanin forms and large amounts of these forms. These findings contribute to a better understanding of bird phenotypic diversity.
\end{abstract}

Keywords: birds, color heterogeneity, melanin-based pigmentation, phylogeny, plumage coloration.

\section{Introduction}

Understanding the mechanics of plumage coloration helps to explain the evolution of bird phenotypic diversity (Hubbard

${ }^{*}$ Corresponding author; email: galvan@ebd.csic.es.

Physiological and Biochemical Zoology 92(3):266-273. 2019. (C) 2019 by The University of Chicago. All rights reserved. 1522-2152/2019/9203-8026\$15.00. DOI: $10.1086 / 702720$ et al. 2010). Some comparative studies of plumage coloration suggest that differences in body size can explain the observed interspecific variability in color heterogeneity (i.e., number of colors; Galván et al. 2013). However, species differ not only in color heterogeneity but also in the level of expression (i.e., intensity) of these colors (Gomez and Théry 2004). In some cases, interspecific differences in plumage color intensity respond to the adaptive benefits of avoiding hybridization between closely related species (Sætre et al. 1997) or are associated with different light environments (McNaught and Owens 2002). Hybridization avoidance, however, cannot represent a general explanation for the interspecific variation in bird plumage color intensity (McNaught and Owens 2002), and it is likely that environmental light is also not a general explanation as derived from its weak association with bird color heterogeneity (Galván et al. 2013).

Instead of (or in addition to) the ecological variables mentioned above, the physiology of plumage color production may provide an explanation for the interspecific variation in color expression levels. A detailed knowledge of these physiological aspects, however, has never been explored in a comparative context of plumage coloration.

Melanins are the most common pigments responsible for the plumage color of birds and are synthesized in specialized cells called melanocytes in a process that comprises physiological limitations, particularly regarding the synthesis of certain chemical forms (Galván and Solano 2016). Therefore, it is likely that producing several forms of melanins, which is required to generate several colors (Galván and Wakamatsu 2016), is more constraining than producing a single or few melanin forms (and a single or few colors) to pigment the plumage. An evolutionary solution to this physiological limitation may consist in producing low amounts of different melanin forms. An economy of pigments in fact represents an explanation for the evolution of melanin-based color traits (Galván and Solano 2009). Such adaptive economy should lead to a negative association between the production of different colors and the level of expression (i.e., intensity) of each color, as variations in the concentration of melanins in feathers are reflected by variations in plumage color intensity (McGraw et al. 2005; Galván and Wakamatsu 2016). We tested this prediction in 96 species of birds breeding in the Iberian Peninsula, showing a large variability in melanin-based plumage color patterns.

\section{Methods}

\section{Bird Species Selection}

Color data of plumage pigmentation corresponding to 96 birds collected in peninsular Spain (table A1) were obtained from museum skins deposited in the bird collection of Doñana Biological 
Station, Consejo Superior de Investigaciones Científicas (Sevilla, Spain). Selected bird species were those present in at least one hundred and fifty $10 \times 10-\mathrm{km}^{2}$ cells in the Universal Transverse Mercator coordinate system of the Spanish sector of the Iberian Peninsula (see Galván et al. 2018). Considering the different color hues of plumage that we could perceive, we observed up to five clearly distinguishable hues in these birds (table A1). Only birds with plumage pigmented by melanins (Galván and Wakamatsu 2016) were considered, excluding those with plumage coloration produced by other pigments, such as carotenoids, or by structural colors. One of the selected species (Petronia petronia) has a small yellow patch produced by carotenoids, but its small size makes its contribution to overall plumage reflectance negligible. Sexually dichromatic species were also excluded because specific values of the analyzed traits cannot be assigned to species in which sexes differ in pigmentation patterns.

\section{Plumage Color Data}

To characterize the pigmentation phenotype of birds, we used two adult specimens of each species (table A1) in which we determined the different color hues that we could perceive and obtained the reflectance spectrum of each of them to quantify the intensity of color expression. Unpigmented plumage areas, bill, and legs were excluded from the analysis. Thus, reflectance measurements were indicative of only melanin-based colored plumage areas.

To obtain the reflectance spectra of the different colors observed in the plumage of birds, we used an Ocean Optics Jaz spectrophotometer (range: $220-1,000 \mathrm{~nm}$ ) with ultraviolet (deuterium) and visible (tungsten-halogen) lamps and a bifurcated $400 \mu \mathrm{m}$ fiber-optic probe. The fiber-optic probe provided both illumination and obtained light reflected from the sample, with a reading area of ca. $1 \mathrm{~mm}^{2}$. All measurements were taken at a $90^{\circ}$ angle to the sample, and reference measurements on a reflectance standard tablet (WS-1, Ocean Optics) were frequently made. For each specimen, five random readings were obtained for each color hue, removing the probe after each measurement. Reflectance curves were determined by calculating the median of the percent reflectance in $10-\mathrm{nm}$ intervals. An average reflectance curve of the five readings for each color hue was then obtained. For each color hue, color intensity was summarized as the summed reflectance across the $300-700 \mathrm{~nm}$ range, with lower values (i.e., darker colors) denoting higher color intensity. Summed reflectance values are thus summed percentages that represent the total amount of reflectance of plumage (Hill and McGraw 2006).

As each color patch covers a different proportion of plumage, the pigmentation of a species cannot be characterized by equally treating the reflectance of different colors. Thus, we weighted the summed reflectance of each hue by the proportion of plumage covered by the corresponding color patch. The total number of pixels of each perceived hue was calculated and then divided by the total number of pixels of the entire plumage to thus obtain the proportion of plumage covered by each color patch. To calculate the pixels covered by each hue, we obtained two digital photographs of each bird specimen, one of the ventral side and one of the dorsal side. The photographs were taken from a fixed distance under standardized light conditions and analyzed with GIMP software (http://www.gimp.org).

\section{Statistical Analyses}

Considering that species are evolutionarily related through a phylogeny, their traits often cannot be treated as independent sample units in statistical analyses (Felsenstein 1985). We obtained 1,000 probable phylogenies for the 96 species used in the study from http://www.birdtree.org, where branch lengths are related to the proportion of nucleotide substitutions among taxa (fig. A1). We obtained the consensus tree of the 1,000 probable phylogenies, applying the $50 \%$ majority rule (i.e., the proportion of a split being present in all trees), and ultrametricized it with a value of 0.1 for the smoothing parameter $\delta$ (Pagel 1999), using the R packages ape (Paradis et al. 2004) and phangorn (Schliep 2011).

However, controlling for phylogenetic effects in the analyses may not be correct under the absence of phylogenetic signal in the studied traits and particularly in the residuals of the model being tested (Garland et al. 2005; Rezende and Finiz-Filho 2012; Symonds and Blomberg 2014). We thus estimated the phylogenetic signal in the studied traits using Blomberg's $K$ statistic (Blomberg et al. 2003). The expression $K=1$ indicates phylogenetic signal in an amount consistent with Brownian motion evolution along the specified phylogeny. If $K>1$, then close relatives are more similar than expected under Brownian motion, whereas if $K<1$, closely related species are less similar than expected under Brownian motion (Blomberg et al. 2003). We estimated Blomberg's $K$ and its significance, which is calculated from a randomization test (Blomberg et al. 2003), using the phylosig command of phytools (Revell 2012). Additionally, we calculated the maximum likelihood value of Pagel's $\lambda$, a multiplier of the off-diagonal elements of the expected variance-covariance matrix (Pagel 1999; Symonds and Blomberg 2014), for the model that was tested to investigate the relationship between variables. Trait evolution follows a Brownian motion model if $\lambda=1$. We thus calculated the maximum likelihood value and 95\% confidence interval for $\lambda$ and tested for $H_{0}(\lambda=0)$ using the pgls command of the caper package (Orme et al. 2013).

We used phylogenetic generalized least squares (PGLS) models to analyze the relationship between plumage summed percentage reflectance (in $\log _{10}$; response variable) and number of colors (in $\log _{10}$; continuous predictor), using the pgls command of caper (Orme et al. 2013). The fitting of PGLS models in caper is conducted considering different branch length modifiers $(\lambda, \kappa$, and $\delta$; Pagel 1999), from which we used $\lambda$. Variables were $\log _{10}$ transformed because this improved the fitting of model residuals to a normal distribution, which was determined by plotting residuals against their expected values given a normal distribution. Body mass could be a confounding variable, as this can explain interspecific variability in plumage color heterogeneity (Galván et al. 2013). Thus, body mass (in $\log _{10}$ ) was included as an additional predictor in the PGLS model. In our PGLS model, we used branch length modifiers calculated through the maximum likelihood estimation of Pagel's $\lambda$. Additionally, we tested the rela- 
Table 1: Summary of fitted models: a phylogenetic generalized least squares (PGLS) model assuming a Brownian motion mode of evolution (PGLS-BM), a PGLS model with a branch length modifier (PGLS-Pagel's $\lambda$ ), and an ordinary least squares (OLS) model

\begin{tabular}{lcccccccc}
\hline & Log likelihood & $\Delta$ AICc & Adj. $R^{2}$ & $F_{2,93}$ & $P$ & $\lambda$ & LB & UB \\
\hline PGLS-Pagel's $\lambda$ & -15.85 & $\ldots$ & .24 & 15.76 & $<.0001$ & .507 & .097 & .866 \\
OLS & -18.93 & 8.33 & .24 & 16.3 & $<.0001$ & $\ldots$ & $\ldots$ & $\ldots$ \\
PGLS-BM & -20.14 & 8.58 & .22 & 14.66 & $<.0001$ & 1.0 & $\ldots$ & $\ldots$ \\
\hline
\end{tabular}

Note. In the PGLS-Pagel's $\lambda$ model, the maximum likelihood value of $\lambda$ and lower and upper bounds (LB and UB) for its $95 \%$ confidence interval are shown. Adj. = adjusted; $\triangle \mathrm{AICc}=$ difference between the corrected Akaike information criterion (AICc) of the model and that of the model with lowest AICc (PGLSPagel's $\lambda$ ).

tionship between plumage summed percentage reflectance, number of colors, and body mass using an ordinary least squares regression (i.e., without considering phylogenetic effects).

\section{Results}

The phylogenetic signal $(K)$ of plumage color traits in the 96 species of birds analyzed was significantly different from zero (plumage summed reflectance: $K=0.84, P=0.021$; number of colors: $K=0.99, P<0.001)$. Consistent with previous studies of many species showing that body size often has $K$ values greater than one (Blomberg et al. 2003), our $K$ value for the body mass of birds was also significantly different from zero and greater than one ( $K=1.89, P<0.001$; see also Galván et al. 2018). The maximum likelihood value of Pagel's $\lambda(0.5)$ in the PGLS model (table 1) was significantly different from zero $(P=0.013)$, thus indicating the existence of phylogenetic signal in an amount consistent with Brownian motion evolution in the relationship between vari- ables. These results indicate the necessity of controlling for phylogenetic effects in the analysis of the relationship between plumage summed reflectance, number of colors, and body mass.

The PGLS model with $\lambda$ was better than the PGLS model without $\lambda$ (i.e., assuming a Brownian model of evolution; $\lambda=1$ ), as indicated by a difference greater than two (8.58) in the corrected Akaike's information criterion between both models (table 1). The PGLS model with $\lambda$ was also better than an ordinary least squares regression model (table 1). The PGLS model with $\lambda$ was therefore the best-fitted model (table 1).

The PGLS model with $\lambda$ was significant $\left(F_{2,93}=15.76\right.$, $P<0.0001)$ and showed a positive and significant effect of the number of colors $(\beta=0.91, P<0.0001)$ and a positive and marginally nonsignificant effect of body mass $(\beta=0.09$, $P=0.088$ ) on plumage summed reflectance. As high-reflectance values correspond to low-intensity colors, this indicates that the intensity of colors decreases with the number of colors and thus that the colors of birds with more colors are lighter than the

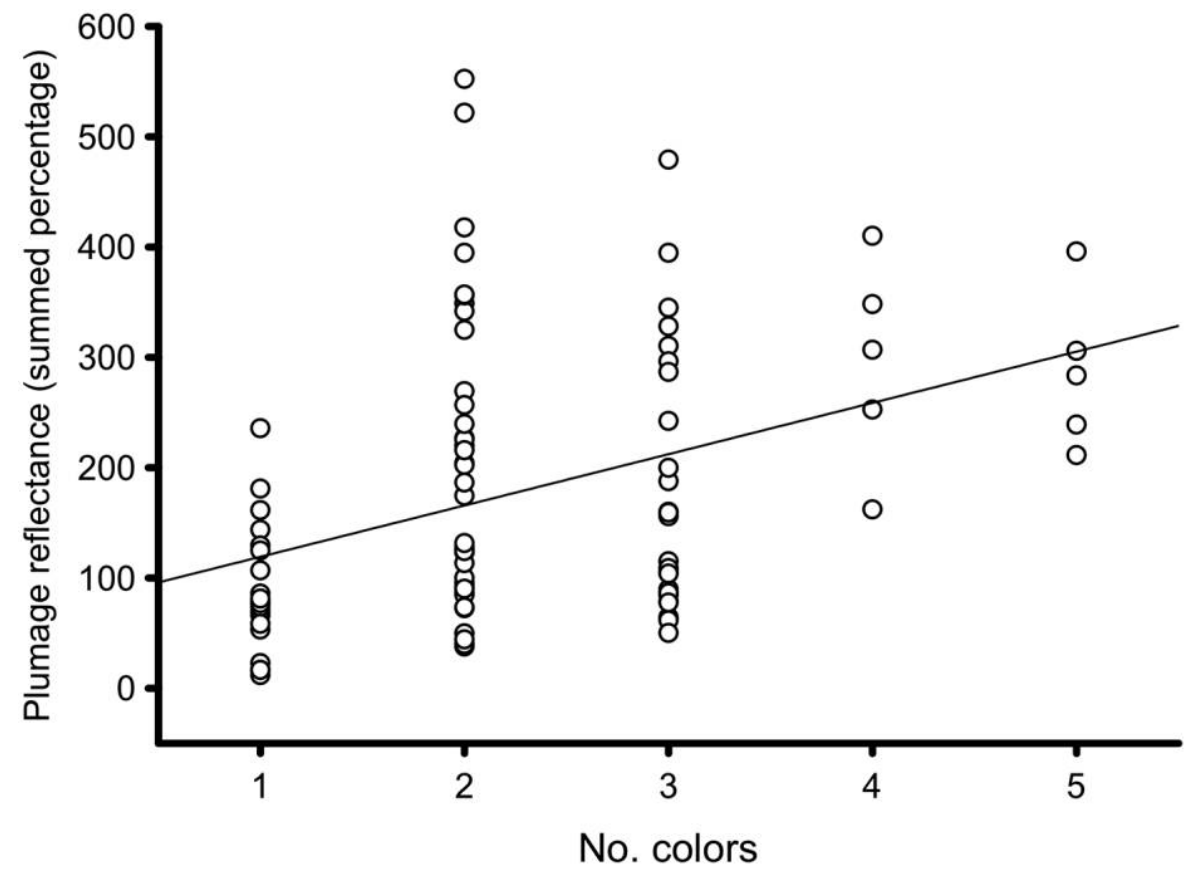

Figure 1. Relationship between melanin-based plumage summed reflectance and number of different colors in 96 species of birds. Higher reflectance values indicate less intense (i.e., lighter) colors. Raw data, not residuals of the phylogenetic generalized least squares model, are shown. The line indicates the ordinary least squares regression line $(r=0.39, n=96, P<0.0001$; slope \pm SE: 46.51 \pm 11.34$)$. 
colors of birds with fewer colors (fig. 1). The same conclusion was obtained using an ordinary least squares regression model (number of colors: $\beta=0.87, P<0.0001$; body mass: $\beta=0.09$, $P=0.034)$.

\section{Discussion}

Birds synthesize melanins in melanocytes located in the skin, which control the type and amounts of melanins that are deposited in the growing feathers (Lin et al. 2013). The diversity of colors that melanins confer to feathers is given by the chemical heterogeneity of these pigments, with the sulphurated forms termed pheomelanins generating lighter colors (yellowish to chestnut) and the nonsulphurated forms termed eumelanins generating darker colors (black, brown, and gray; Galván and Wakamatsu 2016). The synthesis of pheomelanin, however, occurs only when thiols in melanocytes are above a threshold level (Ito and Wakamatsu 2008). As these thiols are composed mainly of a tripeptide that acts as the main intracellular antioxidant (i.e., glutathione) and the free form of its constituent amino acid cysteine, the synthesis of pheomelanin represents the consumption of a valuable antioxidant resource and thus represents a constraining process when antioxidants are required for other vital processes (Napolitano et al. 2014). The production of pheomelanins is therefore more limited in physiological terms than that of eumelanins, and thus not all melanin-based colors are equally favored during the process of melanin synthesis. The existence of a physiological mechanism that maintains cysteine homeostasis in cells (Stipanuk et al. 2009) results in pheomelanin synthesis being favored only under certain physiological and environmental conditions (Galván et al. 2012), as cysteine depletions are not likely to occur.

Given the abovementioned, a production of large amounts of melanins (which leads to an intense color expression) that includes pheomelanins is not expected to represent physiologically favorable conditions. An evolutionary solution to this unviable physiological scenario may consist in producing small amounts of each melanin form when their chemical heterogeneity has been selected to be large (i.e., when the diversity of pheomelanins is included), which may be possible given the independent control of pigmentation exerted by melanocytes (Lin et al. 2013). We hypothesize that this evolutionary solution, which may be favored by natural selection, explains the negative association between melanin-based color heterogeneity and expression that we found in the plumage of 96 species of birds. A similar negative association between the expression of phenotypic traits was reported for song elaboration and plumage ornamentation in a group of birds (Badyaev et al. 2002). However, these authors interpreted their results as the consequence of trade-offs, resolved by birds, between producing different costly traits. We suggest an interpretation of these negative associations based on the incompatibility of biochemical pathways: instead of the result of trade-offs that birds resolve, plumage patterns consisting of colors that are both heterogeneous and intense are not produced because they are hardly possible given the physiological conditions under which melanins are synthesized.

The plumage of birds is one of the most diverse phenotypic traits found in nature, and considering the physiological mechanisms that control the production of pigments has begun to provide a context on which some general rules can be applied to understand how such diversity has evolved and what factors may constrain it (Galván et al. 2017). This study adds a new element to that context, namely, that heterogeneous coloration may be incompatible with intense coloration. Although we focused on melanins, these are the most abundant pigments in birds (Delhey 2015), and therefore these findings are of general validity to understand the evolution of bird phenotypic diversity. Future studies should test for the association found here in taxonomic groups not considered in this study.

\section{Acknowledgments}

Three anonymous reviewers and an editor made comments that improved the manuscript. We thank Rafael Márquez for his help with the spectrophotometric analyses of feathers. Funding support was received from the project CGL2015-67796-P from the Spanish Ministry of Economy, Industry, and Competitiveness (MINECO). I.G. benefited from a Ramón y Cajal Fellowship (RYC-2012-10237) and S.R.-M. from the grant BES2016-077112, both from MINECO. 


\section{APPENDIX}

Table A1: Species names, plumage summed percentage reflectance, number of colors, and body mass for the 96 species of birds included in the study

\begin{tabular}{|c|c|c|c|}
\hline Species & No. colors & Reflectance (summed percentage) & Body mass $(\mathrm{g})$ \\
\hline Accipiter gentilis & 1 & 143.68 & 800 \\
\hline Acrocephalus arundinaceus & 2 & 552.40 & 31 \\
\hline Acrocephalus scirpaceus & 2 & 394.72 & 13 \\
\hline Actitis hypoleucos & 2 & 95.59 & 46 \\
\hline Aegypius monachus & 1 & 129.45 & 10,000 \\
\hline Alauda arvensis & 2 & 348.91 & 45 \\
\hline Alectoris rufa & 5 & 283.58 & 425 \\
\hline Anthus campestris & 2 & 91.61 & 24 \\
\hline Anthus spinoletta & 1 & 66.22 & 22 \\
\hline Anthus trivialis & 2 & 86.42 & 23 \\
\hline Apus apus & 1 & 69.67 & 42 \\
\hline Aquila chrysaetos & 1 & 78.23 & 4,775 \\
\hline Aquila fasciatus & 1 & 53.21 & 2,050 \\
\hline Ardea cinerea & 2 & 220.07 & 900 \\
\hline Ardea purpurea & 3 & 155.97 & 1,000 \\
\hline Asio otus & 3 & 242.41 & 328 \\
\hline Athene noctua & 1 & 77.37 & 170 \\
\hline Bubo bubo & 3 & 328.19 & 2,250 \\
\hline Burhinus oedicnemus & 2 & 341.82 & 410 \\
\hline Buteo buteo & 1 & 235.69 & 875 \\
\hline Caprimulgus europaeus & 3 & 105.19 & 88 \\
\hline Caprimulgus ruficollis & 3 & 115.00 & 75 \\
\hline Certhia brachydactyla & 2 & 84.66 & 10 \\
\hline Cettia cetti & 2 & 226.73 & 14 \\
\hline Charadrius alexandrinus & 3 & 159.95 & 50 \\
\hline Charadrius dubius & 2 & 204.06 & 40 \\
\hline Ciconia ciconia & 1 & 124.79 & 3,250 \\
\hline Ciconia nigra & 1 & 180.94 & 3,000 \\
\hline Cinclus cinclus & 2 & 123.31 & 65 \\
\hline Circaetus gallicus & 1 & 161.40 & 1,750 \\
\hline Cisticola juncidis & 2 & 356.15 & 10 \\
\hline Coccothraustes coccothraustes & 5 & 305.86 & 55 \\
\hline Columba oenas & 4 & 410.18 & 295 \\
\hline Columba palumbus & 4 & 252.67 & 485 \\
\hline Corvus corax & 1 & 73.82 & 1,145 \\
\hline Corvus corone & 1 & 85.82 & 518 \\
\hline Corvus monedula & 2 & 72.52 & 245 \\
\hline Delichon urbicum & 1 & 58.41 & 18 \\
\hline Dryocopus martius & 1 & 106.74 & 325 \\
\hline Elanus caeruleus & 2 & 202.32 & 233 \\
\hline Emberiza cia & 5 & 211.36 & 25 \\
\hline Erithacus rubecula & 2 & 125.73 & 19 \\
\hline Erythropygia galactotes & 3 & 479.27 & 21 \\
\hline Falco peregrinus & 3 & 345.09 & 950 \\
\hline Falco subbuteo & 2 & 225.46 & 235 \\
\hline Fulica atra & 2 & 100.11 & 775 \\
\hline Galerida cristata & 2 & 417.70 & 40 \\
\hline
\end{tabular}


Table A1 (Continued)

\begin{tabular}{|c|c|c|c|}
\hline Species & No. colors & Reflectance (summed percentage) & Body mass $(\mathrm{g})$ \\
\hline Gallinula chloropus & 2 & 49.71 & 330 \\
\hline Glareola pratincola & 5 & 395.98 & 77 \\
\hline Gyps fulvus & 2 & 324.94 & 9,250 \\
\hline Himantopus himantopus & 2 & 37.77 & 180 \\
\hline Hirundo rupestris & 1 & 77.22 & 21 \\
\hline Hirundo rustica & 2 & 40.00 & 21 \\
\hline Jynx torquilla & 3 & 159.29 & 38 \\
\hline Lanius excubitor & 3 & 187.75 & 55 \\
\hline Lanius senator & 2 & 123.96 & 39 \\
\hline Larus cachinnans & 2 & 521.76 & 1,000 \\
\hline Lullula arborea & 2 & 123.27 & 26 \\
\hline Luscinia megarhynchos & 2 & 174.56 & 23 \\
\hline Miliaria calandra & 2 & 73.36 & 47 \\
\hline Milvus milvus & 2 & 269.42 & 1,025 \\
\hline Motacilla alba & 3 & 64.27 & 22 \\
\hline Muscicapa striata & 2 & 113.49 & 16 \\
\hline Neophron percnopterus & 2 & 215.74 & 1,900 \\
\hline Oenanthe hispanica & 2 & 356.96 & 19 \\
\hline Oenanthe leucura & 1 & 11.97 & 41 \\
\hline Otis tarda & 3 & 310.38 & 4,962 \\
\hline Otus scops & 3 & 296.39 & 85 \\
\hline Parus ater & 3 & 89.71 & 9 \\
\hline Parus cristatus & 3 & 61.60 & 12 \\
\hline Passer montanus & 5 & 239.07 & 22 \\
\hline Perdix perdix & 3 & 199.80 & 400 \\
\hline Petronia petronia & 2 & 127.77 & 30 \\
\hline Pica pica & 1 & 22.71 & 178 \\
\hline Podiceps cristatus & 3 & 108.83 & 975 \\
\hline Prunella modularis & 3 & 50.19 & 20 \\
\hline Pterocles alchata & 4 & 306.78 & 243 \\
\hline Pyrrhocorax graculus & 1 & 16.54 & 208 \\
\hline Pyrrhocorax pyrrhocorax & 1 & 16.65 & 320 \\
\hline Rallus aquaticus & 3 & 84.41 & 138 \\
\hline Remiz pendulinus & 4 & 348.25 & 9 \\
\hline Riparia riparia & 2 & 124.33 & 15 \\
\hline Saxicola torquatus & 3 & 103.91 & 16 \\
\hline Scolopax rusticola & 3 & 78.62 & 335 \\
\hline Streptopelia decaocto & 3 & 286.82 & 188 \\
\hline Streptopelia turtur & 4 & 162.18 & 188 \\
\hline Sturnus unicolor & 1 & 81.24 & 90 \\
\hline Sylvia borin & 2 & 256.91 & 20 \\
\hline Sylvia undata & 2 & 90.08 & 11 \\
\hline Tachybaptus ruficollis & 3 & 86.35 & 150 \\
\hline Troglodytes troglodytes & 2 & 186.46 & 11 \\
\hline Turdus philomelos & 2 & 131.59 & 78 \\
\hline Turdus viscivorus & 3 & 77.71 & 125 \\
\hline Tyto alba & 3 & 394.71 & 520 \\
\hline Upupa epops & 2 & 239.61 & 68 \\
\hline Vanellus vanellus & 2 & 44.20 & 230 \\
\hline
\end{tabular}

\footnotetext{
Note. Reflectance refers to the summed percentage values of reflectance across the $300-700 \mathrm{~nm}$ spectral range.
} 


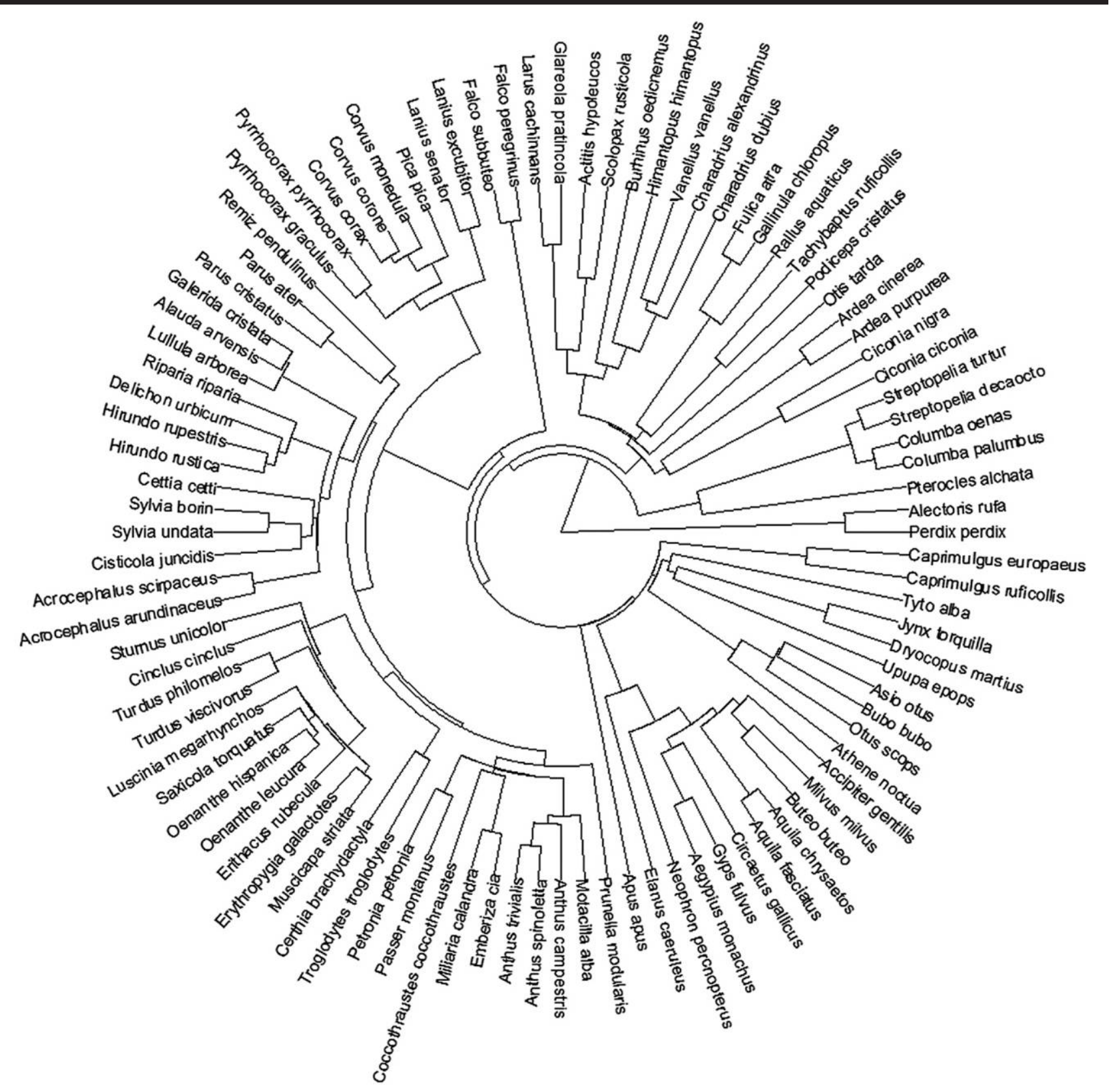

Figure A1. Consensus phylogenetic tree for the 96 species of birds included in the study (Galván et al. 2018) obtained from http://www.birdtree.org. Branch lengths are proportional to nucleotide substitutions. 


\section{Literature Cited}

Badyaev A.V., G.E. Hill, and B.V. Weckworth. 2002. Species divergence in sexually selected traits: increase in song elaboration is related to decrease in plumage ornamentation in finches. Evolution 56:412-419.

Blomberg S.P., T. Garland Jr., and A.R. Ives. 2003. Testing for phylogenetic signal in comparative data: behavioral traits are more labile. Evolution 57:717-745.

Delhey K. 2015. The colour of an avifauna: a quantitative analysis of the colour of Australian birds. Sci Rep 5:1-12.

Felsenstein J. 1985. Phylogenies and the comparative method. Am Nat 125:1-15.

Galván I., J. García-Campa, and J.J. Negro. 2017. Complex plumage patterns can be produced only with the contribution of melanins. Physiol Biochem Zool 90:600-604.

Galván I., G. Ghanem, and A.P. Møller. 2012. Has removal of excess cysteine led to the evolution of pheomelanin? BioEssavs 34:565-568.

Galván I., J.J. Negro, A. Rodríguez, and L.M. Carrascal. 2013. On showy dwarfs and sober giants: body size as a constraint for the evolution of bird plumage colouration. Acta Ornithol 48:65-80.

Galván I., S. Rodríguez-Martínez, and L.M. Carrascal. 2018. Dark pigmentation limits thermal niche position in birds. Funct Ecol 32:1531-1540.

Galván I. and F. Solano. 2009. The evolution of eu- and pheomelanic traits may respond to an economy of pigments related to environmental oxidative stress. Pigment Cell Melanoma Res 22:339-342.

- 2016. Bird integumentary melanins: biosynthesis, forms, function and evolution. Int I Mol Sci 17:520.

Galván I. and K. Wakamatsu. 2016. Color measurement of the animal integument predicts the content of specific melanin forms. RSC Adv 6:79135-79142.

Garland T., Jr., A. F. Bennett, and E. L. Rezende. 2005. Phylogenetic approaches in comparative physiology. IExp Biol 208:3015-3035.

Gomez D. and M. Théry. 2004. Influence of ambient light on the evolution of colour signals: comparative analysis of a Neotropical rainforest bird community. Ecol Lett 7:279-284.

Hill G.E. and K.J. McGraw. 2006. Bird coloration. Vol. 1. Mechanisms and measurement. Harvard University Press, Cambridge, MA.

Hubbard J.K., J.A.C. Uy, M.E. Hauber, H.E. Hoekstra, and R.J. Safran. 2010. Vertebrate pigmentation: from underlying genes to adaptive function. Trends Genet 26:231-239.
Ito S. and K. Wakamatsu. 2008. Chemistry of mixed melanogenesis - pivotal roles of dopaquinone. Photochem Photobiol 84:582-592.

Lin S.J., J. Foley, T.X. Jiang, C.Y. Yeh, P. Wu, A. Foley, C.M. Yen, et al. 2013. Topology of feather melanocyte progenitor niche allows complex pigment patterns to emerge. Science 340:14421445.

McGraw K.J., R.J. Safran, and K. Wakamatsu. 2005. How feather colour reflects its melanin content. Funct Ecol 19:816-821.

McNaught M.K. and I.P. Owens. 2002. Interspecific variation in plumage colour among birds: species recognition or light environment? LEvol Biol 15:505-514.

Napolitano A., L. Panzella, G. Monfrecola, and M. d'Ischia. 2014. Pheomelanin-induced oxidative stress: bright and dark chemistry bridging red hair phenotype and melanoma. Pigment Cell Melanoma Res 27:721-733.

Orme D., R. Freckleton, G. Thomas, T. Petzoldt, S. Fritz, N. Isaac, and W. Pearse. 2013. caper: comparative analyses of phylogenetics and evolution in R. https://CRAN.R-project.org /package $=$ caper.

Pagel M. 1999. Inferring the historical patterns of biological evolution. Nature 401:877-884.

Paradis E., J. Claude, and K. Strimmer. 2004. ape: analyses of phylo-genetics and evolution in $\mathrm{R}$ language. Bioinformatics 20:289-290.

Revell L.J. 2012. phytools: an R package for phylogenetic comparative biology (and other things). Methods Ecol Evol 3:217223.

Rezende E.L. and J.A.F. Diniz-Filho. 2012. Phylogenetic analyses: comparing species to infer adaptations and physiological mechanisms. Compr Physiol 2:639-674.

Sætre G.-P., T. Moum, S. Bures, M. Král, M. Adamjan, and J. Moreno. 1997. A sexually selected character displacement in flycatchers reinforces premating isolation. Nature 387:589592.

Schliep K.P. 2011. phangorn: phylogenetic analysis in R. Bioinformatics 27:592-593.

Stipanuk M.H., I. Ueki, J.E. Dominy Jr., C.R. Simmons, and L.L. Hirschberger. 2009. Cysteine dioxygenase: a robust system for regulation of cellular cysteine levels. Amino Acids 37:5563.

Symonds M.R.E. and S.P. Blomberg. 2014. A primer on phylogenetic generalised least squares. Pp. 105-130 in L.Z. Garamszegi, ed. Modern phylogenetic comparative methods and their application in evolutionary biology. Springer, Heidelberg. 